\title{
ON THE INDEFINITE INTEGRALS OF FUNCTIONS SATISFYING HOMOGENEOUS LINEAR DIFFERENTIAL EQUATIONS ${ }^{1}$
}

\author{
G. M. MULLER
}

1. Introduction. Lommel $[1]^{2}$ established the integral formula

$$
\begin{aligned}
\int z^{\mu} Z_{\nu}(z) d z & =z S_{\mu, \nu}^{\prime}(z) Z_{\nu}(z)-z S_{\mu, \nu}(z) Z_{\nu}^{\prime}(z) \\
& =(\mu+\nu-1) z Z_{\nu}(z) S_{\mu-1, \nu-1}(z)-z Z_{\nu-1}(z) S_{\mu, \nu}(z),
\end{aligned}
$$

where $Z_{\nu}(z)$ is a cylinder function of order $\nu$, and $S_{\mu, \nu}(z)$ is a particular solution of the inhomogeneous differential equation

$$
\left(z^{2} D^{2}+z D+z^{2}-\nu^{2}\right)[v]=z^{\mu+1} .
$$

Other authors have extended his work by considering relations analogous to (1) and (1') for modified Bessel functions [2], with more general functions than $z^{\mu}$ under the integral. Because equation (1) ((1') results from (1) by virtue of the recurrence relations satisfied by $Z_{\nu}$ and $S_{\mu, \nu}$ ) is important both for theoretical [3 ] and computational purposes, it is interesting to ask what sort of relation corresponds to (1) if the left-hand side is replaced by $\int u(z) f(z) d z$ where $u(z)$ is the solution of an arbitrary linear differential equation and $f(z)$ is an arbitrary function.

\section{Integrating vectors. Let}

$$
L \equiv \sum_{i=0}^{n} p_{i} D^{n-i}
$$

be an arbitrary linear differential operator and let $f(z)$ be an arbitrary function (except that the functions $p_{i}(z)$ and $f(z)$ are assumed to be analytic in a given region $R$ of the complex plane and $p_{0}(z) \neq 0$ for $z$ in $R$; these restrictions guarantee that all functions referred to in the theorem of this paper and its proof are analytic in $R$ ). We define an $n$-dimensional function vector $\boldsymbol{h}(\boldsymbol{z})$ to be an integrating vector for the operator $L$ with respect to $f(z)$ if for every solution $u(z)$ of the equation

Received by the editors October 6, 1953 and, in revised form, March 5, 1954.

${ }^{1}$ This work was performed under contract \#W-31-109-Eng-52 with the Atomic Energy Commission.

3 Square brackets refer to the bibliography at the end of this paper. 


$$
L[u]=0
$$

the relation

$$
u(z) f(z)=\frac{d}{d z} \sum_{i=0}^{n} h_{i}(z) u^{(i-1)}(z)
$$

holds. Our principal result is the following

Theorem. A necessary and sufficient condition that $h(z)$ be an integrating vector for $L$ with respect to $f(z)$ is that

$$
h_{i}(z)=-\sum_{j=0}^{n-i}(-1)^{i}\left(p_{n-i-j} v\right)^{(j)}
$$

and $v$ satisfies the equation

$$
L^{*}[v]=f(z),
$$

where $L^{*} \equiv \sum_{i=0}^{n}(-D)^{n-i} p_{i}$ is the Lagrangian adjoint of $L$.

Proof. (a) Sufficiency. Let $h_{i}(z)$ be the linear form in the function $v$ and its derivatives defined by (6). Then Lagrange's identity [4] may be written in the form

$$
v L[u]-u L^{*}[v]=-\frac{d}{d z} \sum_{i=1}^{n} h_{i}(z) u^{(i-1)}(z) .
$$

Evidently, (4) and (7) imply (5).

(b) Necessity. Let $\left\{u_{i}\right\}$ be a complete set of linearly independent solutions of (4), let $u$ be the column vector with components $u_{i}$, and let $U$ be the $n \times n$ matrix whose components are $U_{i j}=u_{i}^{(j-1)}$. A vector $h$ satisfies (5) for every solution of (4) if and only if it satisfies (5) for $u_{i}, i=1, \cdots, n$. Hence the set of all integrating vectors is the set of all solutions $h$ of the $n$th order differential system

$$
\frac{d}{d z} U h=u f(z) .
$$

Now let $\left\{v^{i}\right\}$ be a complete set of linearly independent solutions of $L^{*}[v]=0$ and let $v^{0}$ be a particular solution of (7). Let $h^{j}$ be the vector whose components are given by (6), with $v=v^{j}$. By (a), $\boldsymbol{g}=\boldsymbol{h}^{0}+\sum_{j=1}^{n} c^{j} h^{j}$ (the $c^{j}$ are arbitrary constants) is a solution of (9); since the $h^{i}, j=1, \cdots, n$, are linearly independent, $\delta$ is the general solution of (9).

REMARK 1. By definition, the operator $r L$ ( $r$ an arbitrary function) has the same set of integrating vectors as the operator $L$. The 
necessity condition of the theorem shows that the vector components (6) are independent of $r$.

REMARK 2. For convenience in application, it should be noted that equation (6) may be written in the alternative form

$$
h_{n}(z)=-p_{0} v, \quad h_{i}(z)=-h_{i+1}^{\prime}(z)-p_{n-i} v .
$$

REMARK 3. Let $M$ be the formally self-adjoint second order linear differential operator

$$
M \equiv p D^{2}+p^{\prime} D+q .
$$

The components (10) of the integrating vector then become simply

$$
h_{1}(z)=p v^{\prime}, \quad h_{2}(z)=-p v
$$

where $v$ satisfies the equation

$$
M[v]=f(z) .
$$

The left-hand side of equation (2), after division by $z$, is of the form (11). Equation (1) is thus an immediate consequence of the theorem of this paper.

3. An application. Consider the equation

$$
L[u] \equiv u^{\prime \prime \prime}-\frac{2 \nu}{z} u^{\prime \prime}+\frac{2}{z} u=0 .
$$

This equation is satisfied by the function $\phi_{\nu}(z)[5 ; 6]$, defined on the positive real axis by

$$
\phi_{\nu}(x)=\int_{0}^{\infty} y^{\nu} e^{-y-x / y^{1 / 2}} d y .
$$

We shall use the theorem of this paper to find an expression for integrals of the form $\int \phi_{\nu}(z) z^{\mu} d z$ (with certain restrictions on $\mu$ ).

A particular solution of the equation

$$
L^{*}[v] \equiv-v^{\prime \prime \prime}-2 \nu\left(\frac{v}{z}\right)^{\prime \prime}+2 \frac{v}{z}=z^{\mu}
$$

is given by $v(z)=-z^{\mu+3} \sum_{n=0}^{\infty} a_{n} z^{n}$ if we set

$$
\begin{aligned}
& a_{0}=\frac{1}{(\mu+1)(\mu+2)(\mu+3+2 \nu)}, \quad a_{1}=0, \\
& a_{n}=\frac{2}{(\mu+1+n)(\mu+2+n)(\mu+3+2 \nu+n)} a_{n-2} ;
\end{aligned}
$$


the series exists and is convergent for every finite $z$ provided $\mu$ and $\mu+3+2^{\nu}$ are not negative integers. Using (10), we readily determine the components of an integrating vector,

$$
\begin{gathered}
h_{3}(z)=z^{\mu+3} \sum_{n=0}^{\infty} a_{n} z^{n}, \quad h_{2}(z)=z^{\mu+2} \sum_{n=0}^{\infty} b_{n} z^{n}, \\
h_{1}(z)=z^{\mu+1} \sum_{n=0}^{\infty} c_{n} z^{n}
\end{gathered}
$$

where

$$
\begin{aligned}
& b_{0}=-\frac{1}{(\mu+1)(\mu+2)}, \quad b_{1}=0, \\
& b_{n}=\frac{2}{(\mu+1+n)(\mu+2+n)(\mu+1+2 \nu+n)} b_{n-2}, \\
& c_{0}=\frac{1}{\mu+1}, \quad c_{1}=0 \\
& c_{n}=\frac{2}{(\mu+n)(\mu+1+n)(\mu+1+2 \nu+n)} c_{n-2} .
\end{aligned}
$$

Making use of the fact that $\phi_{\nu}^{\prime}(z)=-\phi_{\nu-1 / 2}(z)$, we finally have

$$
\int \phi_{\nu}(z) z^{\mu} d z=\phi_{\nu}(z) h_{1}(z)-\phi_{\nu-1 / 2}(z) h_{2}(z)+\phi_{\nu-1}(z) h_{3}(z) .
$$

The utility of this expression lies in the fact that the terms of the series (18) are much more readily computed than those obtained by the direct integration of the series for $\phi_{\nu}(z)$ which has a logarithmic singularity at the origin.

\section{BIBLIOGRAPHY}

1. Lommel, Math. Ann. vol. 9 (1876) pp. 425-444.

2. Luke, Journal of Mathematics and Physics vol. 31 (1952) pp. 131-138.

3. Watson, Theory of Bessel functions, $2 \mathrm{~d}$ ed., Cambridge, 1941, pp. 350-351.

4. Ince, Ordinary differential equations, New York, 1944, pp. 123-124.

5. Zahn, Physical Review vol. 52 (1937) pp. 67-71.

6. Laporte, Physical Review vol. 52 (1937) pp. 72-74.

General Electric Company, Richland, Wash. 